\title{
Prevalence and clustering of cardiovascular risk factors in adults in Northeast China
}

\author{
Xin Wang, ${ }^{1,2}$ Fang Yang, ${ }^{1}$ Michiel L Bots, ${ }^{2}$ Weiying Guo, ${ }^{3}$ Di Zhao, ${ }^{1}$ Arno W Hoes, ${ }^{2}$ \\ llonca Vaartjes ${ }^{2}$
}

${ }^{1}$ International Health

Promotion Center, 1st Hospital of Jilin University, Changchun, China

2Julius Center for Health

Sciences and Primary Care, University Medical Center

Utrecht, Utrecht,

The Netherlands

${ }^{3}$ Department of Endocrinology, 1st Hospital of Jilin University, Changchun, China

\section{Correspondence to}

Dr Ilonca Vaartjes, Julius

Center for Health Sciences and

Primary Care, Str 6.131,

University Medical Center

Utrecht, P.O. Box 85500,

Utrecht 3508 GA,

The Netherlands;

c.h.vaartjes@umcutrecht.nl

Received 21 February 2014 Revised 6 June 2014

Accepted 15 August 2014

\begin{abstract}
Aims To estimate the prevalence and clustering of major cardiovascular risk factors in adults in Northeast China.

Methods In total, 37141 individuals undergoing a health screening programme at the International Health Promotion Center at the 1st Hospital of Jilin University were enrolled. Height, weight, blood pressure, fasting serum glucose, total cholesterol (TC), triglyceride (TG), high-density lipoprotein and low-density lipoprotein were recorded.
\end{abstract}

Results Compared with women, the age-standardised prevalence of overweight ( $44.0 \%$ vs $25.0 \%$ ) and obesity ( $20.2 \%$ vs $7.1 \%$ ), hypertension ( $33.7 \%$ vs $19.3 \%$ ), dyslipidaemia (63.8\% vs $42.3 \%$ ), impaired fasting glucose $(5.1 \%$ vs $4.2 \%)$ and diabetes mellitus ( $8.1 \%$ vs $3.5 \%$ ) was higher in men. Overall, $69.1 \%, 32.7 \%$ and $10.0 \%$ of participants had $\geq 1, \geq 2$ and $\geq 3$ major cardiovascular risk factors, respectively (obesity, hypertension, dyslipidaemia, diabetes mellitus). For men, the figures were $79.2 \%, 41.1 \%$ and $13.3 \%$, respectively, and for women $53.4 \%, 19.8 \%$ and $5.0 \%$, respectively. With increasing age and increasing body mass index, the prevalence of clustering of cardiovascular risk factors increased in both men and women.

Conclusions There is a high prevalence of cardiovascular risk factors in adults in Northeast China: one out of three has at least two risk factors. Prevalence was higher in men than in women. Clustering of risk factors is more common with increasing age and body mass index in both men and women. Prevention of the development of risk factors should be an extremely high priority in this region.

\section{INTRODUCTION}

Cardiovascular disease (CVD) is the leading cause of death worldwide, and over $80 \%$ of all deaths from CVD occur in low- and middle-income countries. In China, CVD accounted for $38 \%$ of all deaths in 2010. ${ }^{1}$ It is well known that overweight/ obesity, hypertension, diabetes, dyslipidaemia and tobacco use are primary risk factors for CVD. Owing to currently ongoing demographic, social and economic changes in China, the prevalence of these risk factors for CVD is increasing, which will further increase the burden of CVD. ${ }^{2}$

It has been shown that clustering of cardiovascular risk factors is associated with higher rates of cardiovascular events. ${ }^{3}$ Vice versa, individuals with favourable cardiovascular risk profiles on average live longer and have a better quality of life. ${ }^{4} 5$ Since, many risk factors for CVD are modifiable by specific preventive measures, it is important to quantify the proportion of the population at high risk of CVD to implement targeted interventions to end the CVD epidemic and lower the burden of illness due to CVD.

The area of Northeast China comprising three provinces, Heilongjiang, Jilin and Liaoning, has one of the highest incidences of CVD in China. ${ }^{6} \mathrm{~A}$ recent study on the Chinese 'stroke belt' indicated that nine provincial regions met the criteria for a region of high stroke incidence, and all three provinces of Northeast China were in the belt. ${ }^{7}$ Despite the emergence of CVD, few studies have examined the prevalence and clustering of cardiovascular risk factors in adults in Northeast China. Our study aimed to examine the prevalence of major cardiovascular risk factors, including overweight and obesity, hypertension, diabetes mellitus and dyslipidaemia, and to assess the interdependence of these risk factors in adults in Northeast China.

\section{METHODS}

\section{Study population}

The International Health Promotion Center at the 1st Hospital of Jilin University provides health examinations for residents of Changchun, which is the capital and largest city of Jilin Province, located in the northeast of China. From 2003 to 2010, 37141 individuals who were in a health screening programme at the International Health Promotion Center were enrolled. Participants were mainly current and retired employees of government institutions, universities or companies. Since health screening is considered to be welfare offered by employers, almost all employees participate. Also, as most employees were office workers, men and women were considered to be equally distributed in the labour force. Examinations included anthropometric and blood pressure measurements and blood tests for fasting serum glucose (FSG), total cholesterol (TC), triglyceride (TG), highdensity lipoprotein (HDL) and low-density lipoprotein (LDL). Written informed consent was obtained from each participant. Participants who had conditions newly detected during the examination were referred to the relevant outpatient clinic. The ethics committee of the 1st Hospital of Jilin University approved the protocol for the study.

\section{Blood pressure and anthropometric measurements}

Blood pressure was measured twice with participants in the seated position after $5 \mathrm{~min}$ of rest, using a computerised auto-mercury-sphygmomanometer, Omron BP-203RV IIIC. The mean of these two 
readings was used in the analysis. Participants were advised to avoid cigarette smoking, alcohol and caffeine beverages, and exercise for at least $30 \mathrm{~min}$ before measurement.

Body weight and height were measured with an autoanthropometer, Biospace BSM 330. Height was recorded to the nearest $0.1 \mathrm{~cm}$. Weight was measured with the participant in light indoor clothing without shoes and was recorded to the nearest $0.1 \mathrm{~kg}$.

\section{Laboratory measurements}

Overnight fasting blood samples were collected by venepuncture. Participants who did not meet the overnight fasting criterion were asked to visit the centre later when their fasting time was more than $12 \mathrm{~h}$. Concentrations of TC, TG, HDL and LDL were measured enzymatically on a Hitachi 7600-210 auto analyser (Hitachi, Tokyo, Japan). FSG level was measured on the same autoanalyser. The central laboratory of the 1st Hospital of Jilin University analysed all specimens.

\section{Definitions}

Body mass index (BMI) was calculated as body weight (in $\mathrm{kg}$ ) divided by height (in $\mathrm{m}^{2}$ ). Overweight was defined as $24 \leq \mathrm{BMI}<28 \mathrm{~kg} / \mathrm{m}^{2}$, and obesity as BMI $\geq 28 \mathrm{~kg} / \mathrm{m}^{2}$, based on the Guidelines on Prevention and Control of Overweight and Obesity in Chinese Adults. ${ }^{8}$ Diabetes was defined as an FSG level $\geq 7.0 \mathrm{mmol} / \mathrm{L}$. Impaired fasting glucose (IFG) was defined as $6.1 \leq \mathrm{FSG}<7.0 \mathrm{mmol} / \mathrm{L}$. ${ }^{9}$ Hypertension was defined as systolic blood pressure (SBP) $\geq 140 \mathrm{~mm} \mathrm{Hg}$ or diastolic blood pressure (DBP) $\geq 90 \mathrm{~mm} \mathrm{Hg} .{ }^{10}$ Dyslipidaemia was defined as having 1 or more of the following: TC $\geq 5.18 \mathrm{mmol} / \mathrm{L}, \mathrm{TG} \geq 1.7 \mathrm{mmol} / \mathrm{L}$, $\mathrm{LDL} \geq 3.37 \mathrm{mmol} / \mathrm{L}$ or $\mathrm{HDL} \leq 1.04 \mathrm{mmol} / \mathrm{L} .{ }^{11}$

\section{Statistical analysis}

For continuous variables of cardiovascular risk factors, mean $\pm \mathrm{SD}$ was calculated. For categorical variables, proportions (prevalences) were calculated. To compare prevalence estimates with other studies, we standardised the overall prevalence of cardiovascular risk factors by the direct method to the age distribution of the Chinese population from the 2000 census. Differences in continuous variables were estimated using t tests for independent samples, and prevalence values for categorical variables were compared using Pearson's $\chi^{2}$ test. To calculate the relative risk of having $\geq 1, \geq 2$ and $\geq 3$ cardiovascular risk factors associated with age and sex, we performed multivariable logistic regression, yielding adjusted ORs, as an estimate of the relative risk for having $\geq 1, \geq 2$ and $\geq 3$ risk factors. ORs for sex and age were adjusted for age and sex, respectively. In these analyses, women and those aged 18-29 years served as the respective reference category. A two-tailed $\mathrm{p}$ value $<0.05$ was considered significant. Furthermore, we used $\chi^{2}$ statistics to examine whether obesity was associated with clustering of cardiovascular risk factors. All data were analysed with SPSS V.20.0 for Windows.

\section{RESULTS}

From 2003 to 2010, 37141 individuals (comprising 22549 men and 14592 women) undergoing the health screening programme were enrolled. The mean age of all participants was $45.2 \pm 13.9$ years $(46.1 \pm 14.1$ for men and $43.8 \pm 13.4$ for women; $p>0.05)$. The distribution of major cardiovascular risk factor levels is shown in table 1. Compared with women, men had a higher BMI $\left(25.3\right.$ vs $\left.23.0 \mathrm{~kg} / \mathrm{m}^{2}\right)$, SBP $(125.0$ vs $116.4 \mathrm{~mm} \mathrm{Hg}), \quad$ DBP $(83.1$ vs $76.5 \mathrm{~mm} \mathrm{Hg})$, FSG $(5.5$ vs $5.2 \mathrm{mmol} / \mathrm{L})$, TC (5.0 vs $4.8 \mathrm{mmol} / \mathrm{L})$, TG (2.0 vs $1.3 \mathrm{mmol} / \mathrm{L})$ and LDL (3.0 vs $2.8 \mathrm{mmol} / \mathrm{L})$ and a lower mean HDL (1.4 vs $1.6 \mathrm{mmol} / \mathrm{L})($ all $\mathrm{p}<0.05)$.

In table 2, we present the prevalence of cardiovascular risk factors in both sexes across different age groups and the age-standardised prevalence for those risk factors in men and women. Compared with women, men had a higher age-standardised prevalence of all relevant cardiovascular risk

Table 1 Distribution of cardiovascular risk factors by specific age and gender groups

\begin{tabular}{|c|c|c|c|c|c|}
\hline \multirow[b]{2}{*}{ Risk factor } & \multicolumn{5}{|l|}{ Age group } \\
\hline & $18-29$ & $30-44$ & $45-59$ & $\geq 60$ & Total \\
\hline \multicolumn{6}{|l|}{ Men } \\
\hline $\mathrm{N}$ & 2658 & 8523 & 7590 & 3778 & 22549 \\
\hline $\mathrm{SBP}(\mathrm{mm} \mathrm{Hg})$ & $115.8 \pm 12.6$ & $120.2 \pm 15.0$ & $127.6 \pm 17.7$ & $137.6 \pm 20.1$ & $125.0 \pm 18.0$ \\
\hline $\mathrm{DBP}(\mathrm{mm} \mathrm{Hg})$ & $77.8 \pm 8.7$ & $82.0 \pm 10.9$ & $85.7 \pm 11.7$ & $84.1 \pm 10.2$ & $83.1 \pm 11.1$ \\
\hline FSG (mmol/L) & $5.0 \pm 0.7$ & $5.4 \pm 1.1$ & $5.8 \pm 1.6$ & $5.8 \pm 1.6$ & $5.5 \pm 1.4$ \\
\hline $\mathrm{TC}(\mathrm{mmol} / \mathrm{L})$ & $4.5 \pm 0.9$ & $4.9 \pm 1.0$ & $5.1 \pm 1.0$ & $5.1 \pm 1.0$ & $5.0 \pm 1.0$ \\
\hline $\mathrm{TG}(\mathrm{mmol} / \mathrm{L})$ & $1.5 \pm 1.2$ & $2.2 \pm 2.0$ & $2.1 \pm 1.7$ & $1.6 \pm 1.1$ & $2.0 \pm 1.7$ \\
\hline $\mathrm{HDL}(\mathrm{mmol} / \mathrm{L})$ & $1.4 \pm 0.3$ & $1.4 \pm 0.4$ & $1.4 \pm 0.4$ & $1.3 \pm 0.4$ & $1.4 \pm 0.4$ \\
\hline $\mathrm{LDL}(\mathrm{mmol} / \mathrm{L})$ & $2.7 \pm 0.7$ & $2.9 \pm 0.8$ & $3.1 \pm 0.8$ & $3.1 \pm 0.8$ & $3.0 \pm 0.8$ \\
\hline $\mathrm{BMI}\left(\mathrm{kg} / \mathrm{m}^{2}\right)$ & $24.5 \pm 4.0$ & $25.5 \pm 3.4$ & $25.7 \pm 3.1$ & $24.7 \pm 3.3$ & $25.3 \pm 3.4$ \\
\hline \multicolumn{6}{|l|}{ Women } \\
\hline $\mathrm{N}$ & 2425 & 5563 & 4718 & 1886 & 14592 \\
\hline $\mathrm{SBP}(\mathrm{mm} \mathrm{Hg})$ & $106.4 \pm 11.9$ & $109.3 \pm 13.8$ & $122.6 \pm 18.7$ & $137.2 \pm 20.7$ & $116.4 \pm 19.0$ \\
\hline $\mathrm{DBP}(\mathrm{mm} \mathrm{Hg})$ & $71.0 \pm 7.9$ & $73.7 \pm 9.2$ & $80.3 \pm 11.2$ & $82.6 \pm 10.5$ & $76.5 \pm 10.7$ \\
\hline FSG (mmol/L) & $4.9 \pm 0.6$ & $5.0 \pm 0.6$ & $5.4 \pm 1.2$ & $5.8 \pm 1.7$ & $5.2 \pm 1.0$ \\
\hline $\mathrm{TC}(\mathrm{mmol} / \mathrm{L})$ & $4.3 \pm 0.8$ & $4.5 \pm 0.8$ & $5.1 \pm 1.0$ & $5.5 \pm 1.0$ & $4.8 \pm 1.0$ \\
\hline TG (mmol/L) & $0.9 \pm 0.6$ & $1.1 \pm 0.8$ & $1.5 \pm 1.0$ & $1.8 \pm 1.1$ & $1.3 \pm 0.9$ \\
\hline $\mathrm{HDL}$ (mmol/L) & $1.7 \pm 0.4$ & $1.6 \pm 0.4$ & $1.6 \pm 0.4$ & $1.5 \pm 0.4$ & $1.6 \pm 0.4$ \\
\hline $\mathrm{LDL}(\mathrm{mmol} / \mathrm{L})$ & $2.5 \pm 0.6$ & $2.6 \pm 0.7$ & $3.1 \pm 0.8$ & $3.3 \pm 0.8$ & $2.8 \pm 0.8$ \\
\hline $\mathrm{BMI}\left(\mathrm{kg} / \mathrm{m}^{2}\right)$ & $21.0 \pm 2.8$ & $22.3 \pm 2.9$ & $24.1 \pm 3.0$ & $24.9 \pm 3.4$ & $23.0 \pm 3.3$ \\
\hline
\end{tabular}


Table 2 Prevalence of cardiovascular risk factors in the study population

\begin{tabular}{|c|c|c|c|c|c|c|c|c|c|c|c|}
\hline \multirow[b]{3}{*}{ Risk factor } & \multicolumn{11}{|c|}{ Age group } \\
\hline & \multicolumn{2}{|c|}{$18-29$} & \multicolumn{2}{|c|}{$30-44$} & \multicolumn{2}{|c|}{$45-59$} & \multicolumn{2}{|l|}{$\geq 60$} & \multicolumn{2}{|l|}{ Total } & \multirow[b]{2}{*}{ Age-standardised } \\
\hline & $\mathrm{N}$ & $\%$ & N & $\%$ & N & $\%$ & N & $\%$ & N & $\%$ & \\
\hline Men & 2658 & & 8523 & & 7590 & & 3778 & & 22549 & & \\
\hline Overweight & 893 & 33.6 & 3962 & 46.5 & 3941 & 51.9 & 1743 & 46.1 & 10539 & 46.7 & 44.0 \\
\hline Obesity & 501 & 18.8 & 1900 & 22.3 & 1663 & 21.9 & 578 & 15.3 & 4642 & 20.6 & 20.2 \\
\hline Dyslipidaemia & 1270 & 47.8 & 5831 & 68.4 & 5642 & 74.3 & 2568 & 68.0 & 15311 & 67.9 & 63.8 \\
\hline Hypertension & 402 & 15.1 & 2608 & 30.6 & 3550 & 46.8 & 2219 & 58.7 & 8779 & 38.9 & 33.7 \\
\hline IFG & 60 & 2.3 & 503 & 5.9 & 870 & 11.5 & 481 & 12.7 & 1914 & 8.5 & 5.1 \\
\hline Diabetes & 20 & 0.8 & 386 & 4.5 & 831 & 10.9 & 482 & 12.8 & 1719 & 7.6 & 8.1 \\
\hline Women & 2425 & & 5563 & & 4718 & & 1886 & & 14592 & & \\
\hline Overweight & 242 & 10.0 & 1142 & 20.5 & 1851 & 39.2 & 823 & 43.6 & 4058 & 27.8 & 25.0 \\
\hline Obesity & 50 & 2.1 & 238 & 4.3 & 505 & 10.7 & 336 & 17.8 & 1129 & 7.7 & 7.1 \\
\hline Dyslipidaemia & 495 & 20.4 & 1752 & 31.5 & 2959 & 62.7 & 1494 & 79.2 & 6700 & 45.9 & 42.3 \\
\hline Hypertension & 64 & 2.6 & 482 & 8.7 & 1483 & 31.4 & 1097 & 58.2 & 3126 & 21.4 & 19.3 \\
\hline IFG & 21 & 0.9 & 124 & 2.2 & 290 & 6.1 & 215 & 11.4 & 647 & 4.4 & 4.2 \\
\hline Diabetes & 12 & 0.5 & 53 & 1.0 & 226 & 4.8 & 246 & 13.0 & 537 & 3.7 & 3.5 \\
\hline
\end{tabular}

factors-that is, overweight $(44.0 \%$ vs $25.0 \%)$ and obesity (20.2\% vs $7.1 \%)$, hypertension $(33.7 \%$ vs $19.3 \%)$, dyslipidaemia $(63.8 \%$ vs $42.3 \%)$, IFG $(5.1 \%$ vs $4.2 \%)$ and diabetes mellitus $(8.1 \%$ vs $3.5 \%)$ (all $\mathrm{p}<0.05)$. In men, the prevalence of hypertension and IFG/diabetes increased with age ( $p$ for trend $<0.05$ ), while the prevalence of dyslipidaemia, overweight/ obesity decreased with increasing age. In women, all relevant risk factors increased over the entire age range $(p<0.05)$.

Overall, $20.8 \%$ of the men and $46.6 \%$ of the women in Northeast China did not have any of the studied cardiovascular risk factors (obesity, hypertension, dyslipidaemia or diabetes mellitus; figure 1). In contrast, $79.2 \%, 41.1 \%$ and $13.3 \%$ of men and $53.4 \%, 19.8 \%$ and $5.0 \%$ of women had $\geq 1, \geq 2$ and $\geq 3$ major cardiovascular risk factors, respectively. In total, $69.1 \%, 32.7 \%$ and $10.0 \%$ of participants had $\geq 1, \geq 2$ and $\geq 3$ major cardiovascular risk factors.

The adjusted OR (95\% CI) of having $\geq 1, \geq 2$ and $\geq 3$ cardiovascular risk factors in men compared with women was 3.44 (3.27 to 3.61 ), 2.79 (2.66 to 2.94 ) and 2.80 (2.56 to 3.03), respectively (table 3 ). Moreover, older participants were more likely to have $\geq 1, \geq 2$ and $\geq 3$ cardiovascular risk factors than younger participants (table $3, p<0.05$ for all comparisons).
The percentage of individuals with two or more cardiovascular risk factors clearly increased with increasing BMI in both sexes (figure 2); with increasing BMI, the percentage of men with two or more cardiovascular risk factors increased from $17.6 \%$ in those with BMI of $18.5-24 \mathrm{~kg} / \mathrm{m}^{2}$ to $53.1 \%$ in those with the highest BMI $\left(>28 \mathrm{~kg} / \mathrm{m}^{2}\right)$. Similarly, clustering of two or more cardiovascular risk factors was prominent in women with the highest BMI (46.7\%) as compared with those with the lowest BMI (8.9\%).

\section{DISCUSSION}

CVD is the leading cause of death in China. Within China, the Northeast area has a relatively high incidence of cardiovascular events, ${ }^{6}$ but few studies have examined the CVD risk factors in this area. The present study estimates the prevalence and clustering of major cardiovascular risk factors in a healthy screening population in Northeast China. In this cross-sectional screening of a population of 37141 adults, we found that one out of three has at least two risk factors, and overweight/obesity correlates highly with clustering of other cardiovascular risk factors.

A higher level of clustering of cardiovascular risk factors was also observed in studies in samples from the general Chinese population over the last decade. In 2000 , a nationally representative sample showed that $80.5 \%, 45.9 \%$ and $17.2 \%$ of Chinese
Figure 1 Prevalence of having 0,1 , 2 and $\geq 3$ cardiovascular risk factors in men and women. CRF, cardiovascular risk factors.
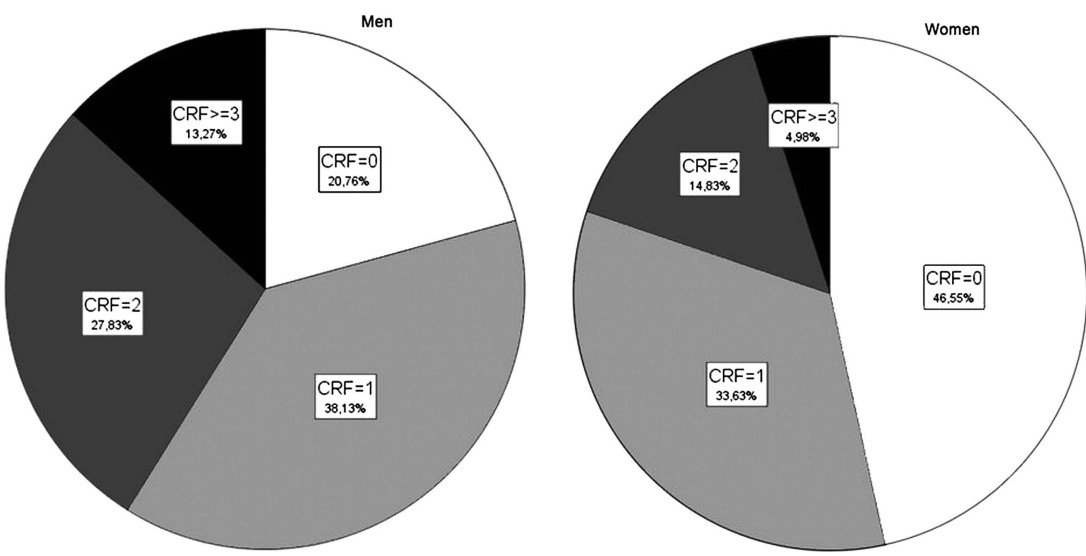
Table 3 Adjusted OR $(95 \% \mathrm{Cl})$ of having $\geq 1, \geq 2$ and $\geq 3$ cardiovascular risk factors associated with sex and age group

\begin{tabular}{clll}
\hline & $\geq 1$ Risk factors & $\geq 2$ Risk factors & $\geq 3$ Risk factors \\
\hline $\begin{array}{c}\text { Age group } \\
18-29\end{array}$ & 1.00 & 1.00 & 1.00 \\
$30-44$ & $2.29(2.14$ to 2.45$)$ & $2.36(2.15$ to 2.60$)$ & $2.18(1.85$ to 2.57$)$ \\
$45-59$ & $6.55(6.08$ to 7.06$)$ & $5.28(4.81$ to 5.81$)$ & $3.95(3.37$ to 4.64$)$ \\
$\geq 60$ & $11.29(10.20$ to 12.50$)$ & $8.47(7.65$ to 9.39$)$ & $4.58(3.87$ to 5.42$)$ \\
Men vs & $3.44(3.27$ to 3.61$)$ & $2.79(2.66$ to 2.94$)$ & $2.80(2.56$ to 3.03$)$ \\
women & & & \\
\hline
\end{tabular}

OR for sex was adjusted for age group, and OR for age group was adjusted for sex. In the logistic regression, women and those aged 18-29 years served as the respective reference category.

adults have $\geq 1, \geq 2$ or $\geq 3$ major cardiovascular risk factors, respectively. ${ }^{12}$ Among 46239 Chinese adults recruited in 20072008 as a nationally representative cohort, the prevalence of having $\geq 2$ risk factors was $55.3 \% .{ }^{13}$ Another national study in 2009-2010 indicated that over half of Chinese adults with cardiovascular risk factors presented with a cluster of risk factors. ${ }^{14}$ Similar prevalent clustering of risk factors was also found in North China, South China ${ }^{15}{ }^{16}$ and Northeast China (as presented in our study).

Clustering of cardiovascular risk factors has also been observed in other countries that have experienced rapid socioeconomic growth like China. Clustering of three or more cardiovascular risk factors was present in $22.7 \%$ of the men and $21.7 \%$ of the women in South Korea. ${ }^{17}$ In Malaysia, 33\% of the national population had two or more risk factors, ${ }^{18}$ and similar findings have been reported for India. ${ }^{19}$

There is ample evidence that the risk of CVD increases substantially with the number of cardiovascular risk factors present. ${ }^{20}$ For example, data from the first National Health and Nutrition Examination Surveys (NHANES) Epidemiologic Follow-up Study in the USA ${ }^{21}$ show that, among participants with one risk factor, more than $50 \%$ of the coronary heart disease and stroke incidence and all-cause mortality was attributable to the presence of this risk factor, while for those with three risk factors, the attributable risk was more than $70 \%$. Furthermore, after 26 years of follow-up, the Chicago Heart Association Detection Project in Industry Study showed that the fewer the risk factors, the higher the quality of life. ${ }^{5}$ Although most of the evidence on the relationship between a worse risk factor profile and a higher number of cardiovascular events comes from established economies, there is ample evidence that risk factor relations as found in westernised societies equally apply to emerging economies. ${ }^{22}$ A recent study identified all three provinces of Northeast China in the stroke belt, all ranking among the top five in terms of stroke incidence rates. ${ }^{7}$

We observed a notable sex difference in cardiovascular riskfactor profiles-that is, the prevalence of cardiovascular risk factors was considerably higher in men than women. Men were three times more likely to have clustering of cardiovascular risk factors than women. According to a national survey on smoking status in 2002, $57.4 \%$ of Chinese men versus $2.6 \%$ of Chinese women were current smokers. ${ }^{23}$ Since we did not include smoking data in our analysis, our observed sex difference in cardiovascular risk-factor profiles might even be an underestimate of the true difference. Our sex-specific findings agree with those reported by others in China. ${ }^{12}$ This sex difference may be due to the fact that Chinese men assume more responsibility in society than women, and Chinese men tend to drink more alcohol and be more susceptible to psychological stress. On the other hand, Chinese women tend to have more awareness of body weight especially in their young and middle years, which could translate into favourable cardiovascular risk profiles. However, this sex difference seems to be inconsistent with data from other countries with fast growing economies. In both South Korea and Malaysia, the prevalence of obesity in women was significantly higher than in men, and furthermore clustering of cardiovascular risk factors was more common in women. ${ }^{17} 24$

Obesity is an independent risk factor for CVD. In China nearly one-quarter of the total 1.3 billion population are overweight. $^{25}$ Our study clearly shows that overweight/obesity is highly related to clustering of other CVD risk factors in adults in Northeast China. This significant relation between clustering of cardiovascular risk factors and obesity is in line with findings from the 2002 China National Nutrition and Health Survey. ${ }^{26}$ Furthermore, rates of metabolic syndrome have been found to be high among obese school children in China. ${ }^{27} \mathrm{~A}$ recent pooled analysis of 97 prospective cohorts suggests that a reduction in blood pressure, cholesterol and glucose might counteract about half of the excess risk of coronary heart disease and threequarters of the excess risk of stroke associated with high BMI. ${ }^{28}$ Taken together, these results indicate that efforts to promote a
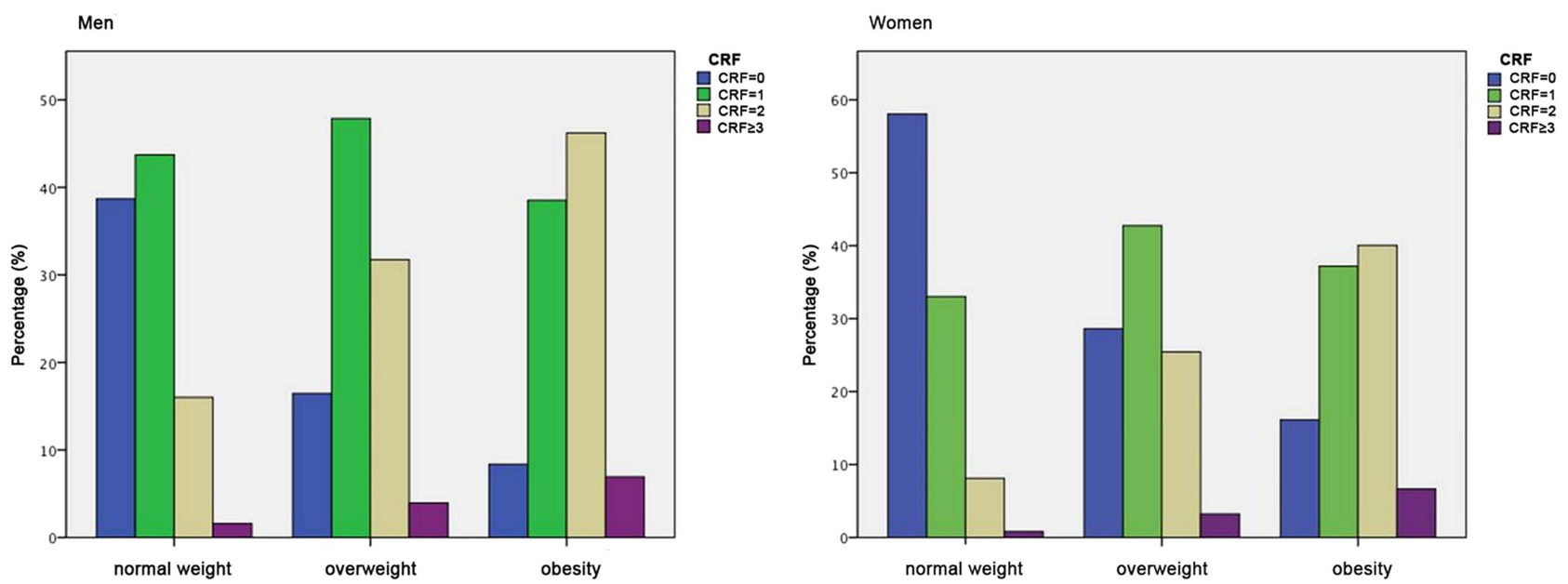

Figure 2 Prevalence of clustering of cardiovascular risk factors by distribution of BMI in men and women. CRF, cardiovascular risk factors; BMI, body mass index. Normal weight, $18 \leq \mathrm{BMl}<24 \mathrm{~kg} / \mathrm{m}^{2}$; overweight, $24 \leq \mathrm{BMl}<28 \mathrm{~kg} / \mathrm{m}^{2}$; obesity, BMl $\geq 28 \mathrm{~kg} / \mathrm{m}^{2}$. 
healthy diet and increase physical activity to maintain optimum weight must be a national priority.

China is undergoing fast economic transition, with rapid changes in urbanisation and industrialisation such as many developed countries have experienced since the 1960s. There is no doubt that the changes in behaviour and lifestyle due to this transition result in increased prevalence of cardiovascular risk factors. In the USA, hypertension prevalence peaked at $36.3 \%$ in NHANES I (1971-1974) and declined to $20.4 \%$ in NHANES III (1988-1991). ${ }^{29}$ In China, an increase in prevalence of hypertension has been observed, from $\sim 18 \%$ in 2002 to $25 \%$ in 2007 and $33 \%$ in $2010,{ }^{30} 31$ with little evidence of a decline. The Framingham Heart Study found a doubling of the incidence of type 2 diabetes over 30 years from the 1970s to the 1990s. ${ }^{32}$ In a 2010 cross-sectional survey in China, the prevalence of diabetes was $11.6 \%$, whereas the prevalence of prediabetes (ie, increased risk of diabetes) was $50 \%{ }^{33}$ Studies in developed countries since the 1960s showed that coronary heart disease mortality has fallen $\sim 50 \%$ due to risk-factor reductions such as reductions in cigarette smoking, blood pressure and cholesterol levels. ${ }^{34}$ This 'rise and fall' pattern of CVD epidemics in developed countries offers potential lessons for achieving similar results in developing countries such as China.

A major strength of our study is the fact that its results are based on findings in a large sample of the Chinese adult population and that it is the first study to provide scientific evidence on the prevalence and clustering of cardiovascular risk factors in adults in Northeast China, an area with a relatively high incidence of CVD. A limitation of the study is that we did not have smoking data in our analysis, which might underestimate the prevalence and clustering of cardiovascular risk factors, especially in men, given that $\sim 60 \%$ of Chinese men are current smokers. ${ }^{23}$ Thus, the cardiovascular risk profile is even worse than we observed, especially among men. Another potential limitation is the representativeness of our study participants, who were employees undergoing a routine health check paid for by their company or institution. It may be that this study population represents a higher socioeconomic and nutritional status and tends to have a more unfavourable cardiovascular risk profile than a population from the lower socioeconomic classes. Hence, the prevalence estimates in our study might not represent the true prevalence of the risk factors in Northeast China.

In conclusion, our study shows that there is a high prevalence of modifiable cardiovascular risk factors in adults in Northeast China: one out of three has at least two risk factors. In addition, our data confirm a significant association between overweight/ obesity and clustering of cardiovascular risk factors. Therefore, comprehensive population-based and high-risk individual interventions must be developed and implemented to improve the cardiovascular health status in Northeast China.

Contributors XW, FY, MLB and IV conceived and designed the study. XW performed the data analyses and wrote the first draft of the manuscript. AWH, WG and DZ commented on the study. All authors read, provided input for, and approved the final draft of the manuscript.

\section{Competing interests None.}

Patient consent Obtained.

Ethics approval Ethics committee of the 1st Hospital of Jilin University, Changchun, China.

Provenance and peer review Not commissioned; externally peer reviewed.

\section{REFERENCES}

Word Health Organization. Word Health Organization, 2010. China. 2013.

2 World Health Organization. Cardiovascular disease. 2014.
3 Malik S, Wong ND, Franklin SS, et al. Impact of the metabolic syndrome on mortality from coronary heart disease, cardiovascular disease, and all causes in United States adults. Circulation 2004:110:1245-50.

4 Stamler J, Stamler R, Neaton JD, et al. Low risk-factor profile and long-term cardiovascular and noncardiovascular mortality and life expectancy: findings for 5 large cohorts of young adult and middle-aged men and women. JAMA 1999:282:2012-18.

5 Daviglus ML, Liu K, Pirzada A, et al. Favorable cardiovascular risk profile in middle age and health-related quality of life in older age. Arch Intern Med 2003:163:2460-8.

6 Group of monitoring trends and determinants in cardiovascular disease (MONICA) in 16 provinces in China. Epidemiology of acute coronary heart events in 16 provinces in china. 1993;14:10-13.

$7 \mathrm{Xu} \mathrm{G}, \mathrm{Ma}$ M, Liu X, et al. Is there a stroke belt in china and why? Stroke 2013;44:1775-83.

8 Chen CM, Kong LZ. The prevention and control guide of Chinese adults overweight and obesity. Beijing: People's Medical Publishing House, 2006.

9 Expert Committee on the Diagnosis and Classification of Diabetes Mellitus. Report of the expert committee on the diagnosis and classification of diabetes mellitus. Diabetes Care 2000;23(Suppl 1):S4-19.

10 [No authors listed]. The fifth report of the joint national committee on detection, evaluation, and treatment of high blood pressure (JNC V). Arch Intern Med 1993:153:154-83.

11 Expert Committee on the Prevention and Control of Dyslipidemia in Chinese Adults. Guidelines on prevention and control of dyslipidemia in chinese adults. Chin J Cardiol 2007;35:5

12 Gu D, Gupta A, Muntner P, et al. Prevalence of cardiovascular disease risk factor clustering among the adult population of china: results from the international collaborative study of cardiovascular disease in Asia (InterAsia). Circulation 2005;112:658-65.

13 Yang ZJ, Liu J, Ge JP, et al. Prevalence of cardiovascular disease risk factor in the Chinese population: the 2007-2008 china national diabetes and metabolic disorders study. Eur Heart J 2012;33:213-20.

14 Gao B, Zhang L, Wang $\mathrm{H}$, et al. Clustering of major cardiovascular risk factors and the association with unhealthy lifestyles in the Chinese adult population. PLOS ONE 2013;8:e66780.

15 Lao XQ, Zhang YH, Wong MC, et al. The prevalence of metabolic syndrome and cardiovascular risk factors in adults in Southern China. BMC Public Health 2012:12:64.

16 Zhang L, Qin LQ, Cui HY, et al. Prevalence of cardiovascular risk factors clustering among suburban residents in Beijing, China. Int J Cardiol 2011;151:46-9.

17 Park HS, Yun YS, Park JY, et al. Obesity, abdominal obesity, and clustering of cardiovascular risk factors in South Korea. Asia Pac J Clin Nutr 2003;12:411-18.

18 Selvarajah S, Haniff J, Kaur G, et al. Clustering of cardiovascular risk factors in a middle-income country: a call for urgency. Eur J Prev Cardiol 2013:20:368-75.

19 Gupta S, Gudapati R, Gaurav K, et al. Emerging risk factors for cardiovascular diseases: Indian context. Indian J Endocrinol Metab 2013;17:806-14.

20 Ji J, Pan E, Li J, et al. Classical risk factors of cardiovascular disease among Chinese male steel workers: a prospective cohort study for 20 years. BMC Public Health 2011;11:497.

21 Yusuf HR, Giles WH, Croft JB, et al. Impact of multiple risk factor profiles on determining cardiovascular disease risk. Prev Med 1998;27:1-9.

22 Woodward M, Huxley $\mathrm{H}$, Lam TH, et al. A comparison of the associations between risk factors and cardiovascular disease in Asia and Australasia. Eur J Cardiovasc Prev Rehabil 2005;12:484-91.

23 Yang GH, Ma JM, Liu N. Smoking and passive smoking in Chinese population. Zhonghua Liu Xing Bing Xue Za Zhi 2005;26:77-83.

24 Wagner A, Arveiler D, Ruidavets JB, et al. Gender- and age-specific trends in coronary heart disease mortality in France from 2000 to 2007: results from the MONICA registers. Eur J Prev Cardiol 2014;21:117-22.

25 Wu YF, Ma GS, Hu YH, et al. The current prevalence status of body overweight and obesity in China: data from the China national nutrition and health survey. Zhonghua Yu Fang Yi Xue Za Zhi 2005;39:316-20.

26 Du SM, Ma GS, Li YP, et al. Relationship of body mass index, waist circumference and cardiovascular risk factors in Chinese adult. Biomed Environ Sci 2010;23:92-101.

27 Liu W, Lin R, Liu A, et al. Prevalence and association between obesity and metabolic syndrome among Chinese elementary school children: a school-based survey. BMC Public Health 2010;10:780.

28 The Global Burden of Metabolic Risk Factors for Chronic Diseases Collaboration (BMI Mediated Effects). Metabolic mediators of the effects of body-mass index, overweight, and obesity on coronary heart disease and stroke: a pooled analysis of 97 prospective cohorts with 1.8 million participants. Lancet 2014;383:970-83.

29 Burt VL, Cutler JA, Higgins M, et al. Trends in the prevalence, awareness, treatment, and control of hypertension in the adult US population. Data from the health examination surveys, 1960 to 1991. Hypertension 1995;26:60-9.

30 Wu Y, Huxley R, Li L, et al. Prevalence, awareness, treatment, and control of hypertension in China: data from the China national nutrition and health survey 2002. Circulation 2008;118:2679-86. 
31 Li YC, Wang LM, Jiang Y, et al. Prevalence of hypertension among Chinese adults in 2010. Zhonghua Yu Fang Yi Xue Za Zhi 2012:46:409-13.

32 Fox CS, Pencina MJ, Meigs JB, et al. Trends in the incidence of type 2 diabetes mellitus from the 1970s to the 1990s: the Framingham heart study. Circulation 2006;113:2914-18
33 Xu Y, Wang L, He J, et al. Prevalence and control of diabetes in Chinese adults. JAMA 2013;310:948-59.

34 Hardoon SL, Whincup PH, Lennon LT, et al. How much of the recent decline in the incidence of myocardial infarction in British men can be explained by changes in cardiovascular risk factors? Evidence from a prospective population-based study. Circulation 2008;117:598-604. 